\title{
Long-Term Outcomes of Selective Laser Trabeculoplasty (SLT) Treatment
}

\author{
Marcelo Ayala* and Enping Chen
}

Glaucoma Department, St. Erik Eye Hospital, Karolinska Institutet, Stockholm, Sweden

\begin{abstract}
Purpose: Glaucoma is a progressive optic neuropathy that may lead to blindness. Reducing intraocular pressure (IOP) seems to be the only treatment that slows progression in glaucoma. IOP can be decreased by pharmaceutical treatment, laser treatment or surgery.

The aim of the present study was to assess the long-term efficacy of selective laser trabeculoplasty (SLT) treatment.

Methods: Retrospective chart review of eyes that underwent SLT between 1 January 2005 and 31 December 2005. The primary outcome measure was time to failure after SLT treatment. Failure after SLT was defined as any one or more of the following: change in the medical treatment, performance of a further SLT treatment, the patient being sent for surgery. All patients were treated over $90^{\circ}$ with SLT.

Results: 120 eyes of 120 patients were identified. The average time to failure after SLT was 18 months. The success rate after 12 months was $62 \%$, after 24 months $34 \%$, after 36 months $28 \%$ and after 48 months $24 \%$.

Conclusions: The long-term effects of SLT when eyes were treated over $90^{\circ}$ seem to be low. The authors recommend treating patients over $180^{\circ}$, as has traditionally been done. We suggest that this will improve the long-term results.
\end{abstract}

Keywords: Glaucoma, laser, intraocular pressure.

\section{INTRODUCTION}

Glaucoma is a progressive neuropathy localized in the optic nerve that may lead to blindness. Reducing intraocular pressure (IOP) seems to be the only treatment that slows progression in glaucoma. There are several methods to reduce IOP: pharmaceutical treatment, laser treatment and surgery. In the Glaucoma Laser Trial Follow-up study, after 7 years of follow-up patients who had undergone argon laser trabeculoplasty (ALT) had lower IOP than patients taking drugs $[1,2]$. However, in selective laser trabeculoplasty (SLT) there is still not sufficient evidence to determine the effectiveness of laser trabeculoplasty compared to medication. Laser trabeculoplasty appears to be less costly than current pharmaceutical treatment [3].

Argon laser trabeculoplasty (ALT) was introduced about 25 years ago and involves the use of a blue/green argon laser (major wavelength peaks at $488 \mathrm{~nm}$ and $514 \mathrm{~nm}$ ) to treat the outer part of the trabecular meshwork and thereby improve aqueous flow and thus reduce IOP. Selective Laser Trabeculoplasty (SLT) is a rather new treatment alternative. It involves using a frequency-doubled q-switched Nd:YAG laser $(532 \mathrm{~nm})$ that delivers a low-energy, large-spot, very brief pulse to selectively target cells of the trabecular meshwork. This "gentler" laser application is thought to stimulate the pigmented trabecular meshwork cells and thus facilitate improved aqueous outflow. SLT treatment may hold clinical advantages over ALT treatment.

Long-term follow up after SLT treatment has been reported by Gracner T et al. [4] and shows a success rate of

*Address correspondence to this author at the St. Erik Eye Hospital, Polhemsgatan 50, SE-112 82 Stockholm, Sweden; Tel: +46-8-672 3507; Fax: +46-8-672 3325; E-mail: marcelo.ayala@sankterik.se
$58 \%$ after 48 months. Weinand FS \& Althen F [5] showed a 4 -year success rate of $44 \%$ after SLT treatment. Juzych et al. [6] in a retrospective study comparing ALT with SLT reported a 4-year success rate of $32 \%$ after SLT treatment. In all the above mentioned studies 180 degrees of the trabecular meshwork was treated.

The aim of the present study was to determine whether the long-term (4 years) efficacy of SLT treatment was maintained when only 90 degrees of the trabecular meshwork was treated.

\section{MATERIALS AND METHODS}

This was a retrospective chart review of patients treated from $1^{\text {st }}$ January 2005 till $31^{\text {st }}$ December 2005 . Inclusion criteria were open-angle glaucoma (primary open angle, pseudoexfoliation or pigmentary glaucoma) and ocular hypertensive (OHT) patients, older than 18 years, who were uncontrolled on pharmaceutical therapy and who had been given SLT treatment. Exclusion criteria were patients who could not be followed for at least 4 years.

Data recorded from each patient included age, sex, type of glaucoma, glaucoma medications and the SLT protocol (number of spots and laser power settings). The IOP values before and after SLT were also registered. All patients continued with the same pharmaceutical treatment after SLT.

The SLT protocol consisted of treatment that was performed in $90^{\circ}$ with the SLT Solo laser (Ellex, Mainhausen, Germany) using between 25-30 spots applied to the trabecular meshwork. The energy level ranged from 0.6 to $1.0 \mathrm{~mJ}$. All patients were treated by the same physician (E.C.). Apraclonidine was instilled once after SLT treatment. Follow-up IOP measurements were obtained at least 1, 6, 12, 24 and 48 months after SLT treatment. 
The main outcome variable analysed was the time to failure after SLT treatment. Failure after SLT was defined as any one or more of the following: change in the pharmaceutical treatment, performance of a further SLT treatment, the patient being sent for surgery.

When two eyes of the same patient were treated, one eye was chosen at random for analysis.

\section{Statistical Analysis}

All data were entered into a database. Success rates were determined with the Kaplan-Meier survival analysis. Statistical software: STATA (Statacorp, 4905 Lakeway Drive, College Station, Texas 77845, USA).

\section{RESULTS}

In all, 120 eyes of 120 patients were identified during the defined time period. The baseline characteristics of the sample are given in Table 1.

Table 1. Base-Line Characteristics of the Subjects Included in the Study

\begin{tabular}{|c|l|}
\hline \multicolumn{1}{|c|}{ Characteristic } & \\
\hline \hline Age (SD) & $76.5(11.8)$ years \\
\hline Gender & $75 \%$ women \\
\hline IOP before SLT (mmHg) (SD) & $24.7(4.6)$ \\
\hline $\begin{array}{c}\text { Number of SLT procedures undergone: } \\
\text { SLT 1 (\%) }\end{array}$ & $74(62)$ \\
SLT 2 (\%) & $38(32)$ \\
SLT 3 (\%) & $5(4)$ \\
SLT 4 (\%) & $3(2)$ \\
\hline Average number of spots per eye (SD) & $26.9(4.8)$ \\
\hline Average dose per eye (mJ) (SD) & $0.88(0.11)$ \\
\hline Average anterior chamber (0-4) (SD) & $3.8(0.49)$ \\
\hline Average pigmentation (0-3) & $0.77(0.66)$ \\
\hline $\begin{array}{r}\text { Diagnosis } \\
\text { Exfoliative glaucoma (\%) } \\
\text { Primary open-angle glaucoma (\%) } \\
\text { Ocular hypertension (\%) }\end{array}$ & $73(61)$ \\
Pigmentary glaucoma (\%) & $9(7.5)$ \\
\hline Number of medications (n) (SD) & $1.65(0.83)$ \\
\hline
\end{tabular}

The average time to failure after SLT was 18 months. Success rate of SLT is shown in Fig. (1).

Intraocular pressure (IOP) values are shown in Fig. (2). The registered IOP was until the eye was classified as "failure".

\section{DISCUSSION}

Selective laser trabeculoplasty is a commonly accepted therapy for lowering IOP. The present study aims to answer the question of the efficiency and duration of IOP reduction after SLT over a longer period of time. Long-term follow up after SLT treatment has been reported by Gracner T et al. [4] showing a success rate of $58 \%$ after 48 months. Weinand FS $\&$ Althen F [5] showed a 4-year success rate of $44 \%$ after SLT treatment. Juzych et al. [6] in a retrospective study comparing ALT with SLT reported a 4-year success rate of $32 \%$ after SLT treatment. In our study, the 4-year success rate was $24 \%$. This low success rate may have one of several explanations. First, the definition of "success" chosen in our study was very strict, thus shortening the time to failure. Second, patients were treated over $90^{\circ}$, and this meant that the total dose and effect were lower than those in the studies presented above.

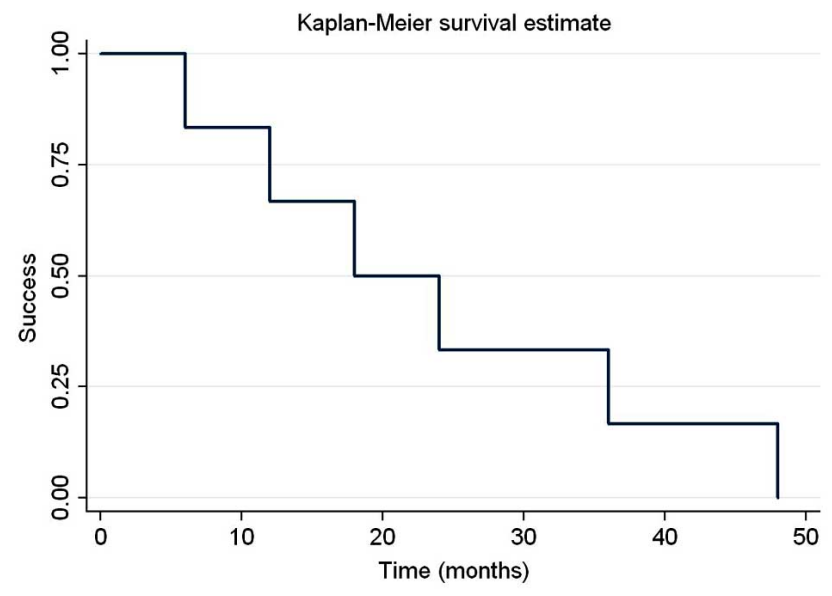

Fig. (1). Kaplan-Meier survival analysis.

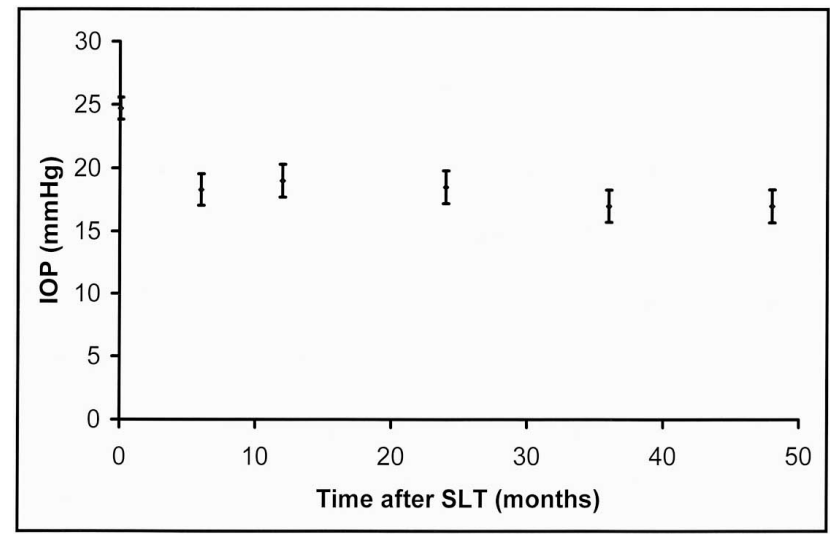

Fig. (2). IOP measurements. Note that the amount of included eyes was not the same in the different time points: 6 months $(n=82), 12$ months $(\mathrm{n}=43), 24$ months $(\mathrm{n}=27), 36$ months $(\mathrm{n}=23), 48$ months $(\mathrm{n}=20)$. Eyes were excluded when they were defined as "failure".

In our study, the probability of success one year after SLT was $62 \%$. Song et al. [7] showed a very low success rate one year after SLT treatment of $15.8 \%$ treating over $180^{\circ}$, but the population studied had an extremely low preoperative average intraocular pressure $(17.6 \mathrm{mmHg})$, making these results difficult to compare. Weinand FS \& Althen F [5], treating over $180^{\circ}$ of the trabecular meshwork, showed a one-year success rate of $60 \%$, comparable to the value we observed. This means that SLT treatment over $90^{\circ}$ probably gives a similar IOP reduction as treating over $180^{\circ}$, but this is only true at one year after SLT. The 4-year success rate showed by Weinand FS \& Althen F was $44 \%$, nearly double than the success rate found in this study 
(24\%). Long-term IOP reduction seems to be better when laser treatment is delivered over $180^{\circ}$.

The reason patients were treated only over $90^{\circ}$ was a study published by Chen et al. [8] showing similar IOP reduction with SLT over $90^{\circ}$ as that achieved when SLT was delivered over $180^{\circ}$. The authors evaluated the IOP reduction at 1, 4, and 7 months after treatment. However, Nagar M et al. [9] showed a one-year success rate of $34 \%$ when eyes were treated over $90^{\circ}$, while the one-year success rate was $65 \%$ when the eyes were treated over $180^{\circ}$. Comparing $180^{\circ}$ and $360^{\circ}$ the authors found an increased success rate, but the difference against treating $180^{\circ}$ was not significant. The authors concluded that success rates motivate treating $360^{\circ}$ of the angle at each laser session, rather than just $180^{\circ}$.

Our study is limited by its retrospective design. One issue to be considered is selection bias. All patients were treated at a tertiary referral centre where the patient population tends to have more advanced glaucoma. The severity of glaucoma may have been a factor, and this was not directly evaluated in this study. Another possible selection bias is the quite high percentage of pseudoexfoliative glaucoma patients included in the study (61\%). Gracner et al. [10] showed no difference in SLT results comparing capsular glaucoma and primary open-angle glaucoma. The authors evaluated the short-term results for an average of 12 months. It is very possible that SLT results in the long term (4 years) are different from those in the short term (1 year). Pseudoexfoliative glaucoma patients probably require more treatment, and their inclusion in this study has increased the failure rate.

In conclusion, long-term IOP reduction after SLT seems not to hold when eyes are treated over $90^{\circ}$. The authors recommend treating patients over $180^{\circ}$, as has traditionally been done. We suggest that this will improve the long-term results.

\section{DISCLOSURE}

1) No commercial interests.

2) Supported in part by a grant from the Karolinska Institutet's Foundation.
3) Informed consent: the study was performed with informed consent and following all guidelines for experimental investigations required by the Karolinska Institutet Ethics Committee.

\section{REFERENCES}

[1] The Glaucoma Laser Trial Research Group, The Glaucoma Laser Trial (GLT) 2. Results of argon laser trabeculoplasty versus topical medicines. Ophthalmology 1990; 97: 1403-13.

[2] The Glaucoma Laser Trial Research Group. The Glaucoma Laser Trial (GLT) and glaucoma laser trial follow-up study: 7. Results. Am J Ophthalmol 1995; 120: 718-31.

[3] Lee R, Hutnik CM. Projected cost comparison of selective laser trabeculoplasty versus glaucoma medication in the Ontario Health Insurance Plan. Can J Ophthalmol 2006; 41: 449-56.

[4] Gracner T, Falez M, Gracner B, Pahor D. Long-term follow-up of selective laser trabeculoplasty in primary open-angle glaucoma. Klin Monatsbl Augenheilkd 2006; 223: 743-47.

[5] Weinand FS, Althen F. Long-term clinical results of selective laser trabeculoplasty in the treatment of primary open angle glaucoma. Eur J Ophthalmol 2006; 16: 100-4.

[6] Juzych MS, Chopra V, Banitt MR, et al. Comparison of long-term outcomes of selective laser trabeculoplasty versus argon laser trabeculoplasty in open-angle glaucoma. Ophthalmology 2004; 111: 1853-1859.

[7] Song J, Lee PP, Epstein DL, et al. High failure rate associated with 180 degrees selective laser trabeculoplasty. J Glaucoma 2005; 14 : 400-408.

[8] Chen E, Golchin S, Blomdahl S. A comparison between 90 degrees and 180 degrees selective laser trabeculoplasty. J Glaucoma 2004 13: 62-65.

[9] Nagar M, Ogunyomade A, O'Brart DP, Howes F, Marshall J. A randomised, prospective study comparing selective laser trabeculoplasty with latanoprost for the control of intraocular pressure in ocular hypertension and open angle glaucoma. $\mathrm{Br} \mathrm{J}$ Ophthalmol 2005; 89: 1413-17.

[10] Gracner T. Intraocular pressure response of capsular glaucoma and primary open-angle glaucoma to selective Nd:YAG laser trabeculoplasty: a prospective, comparative clinical trial. Eur J Ophthalmol 2002; 12: 287-92. 